expression of total eNOS, p-eNOSS1177, total PP2A, and p-PP2AY307. For activity p-eNOSS1177/total eNOS and p-PP2AY307/ total PP2A ratio was used. A two-way ANOVA was used for statistical analysis. RESULTS/ANTICIPATED RESULTS: Irrespective of the donors' race, there was no influence of serum treatment or interaction effect in any of the measured proteins of interest. Moreover, compared to CA, HUVECs from AA had lower expression of eNOS irrespective of condition (race $\mathrm{p}=0.01$ ). Compared to CA, HUVECs from AA tended to have lower expression of p-eNOSS1177 irrespective of condition (race $\mathrm{p}=0.07$ ). However, there was no racial differences in eNOS activity $(\mathrm{p}=0.68)$. There was no racial difference in the expression of PP2A $(\mathrm{p}=0.35)$, p-PP2AY307 $(\mathrm{p}=0.30)$, or PP2A activity $(\mathrm{p}=0.97)$ in all conditions. DISCUSSION/SIGNIFICANCE OF FINDINGS: Our preliminary results suggest no influence serum constituents from hypertensive donors or race on PP2A or eNOS expression and activity in HUVECS. Future research should consider conducting proteomics profiling to compare NT and HT serum.

39800

\section{Immune Checkpoint Blockade during Periprosthetic Joint Infection}

Shay Warren, Greg Charville and *Derek Amanatullah Stanford University

ABSTRACT IMPACT: If immune checkpoint blockade increases bacterial clearance with or without antibiotics in vitro, clinical application would be almost immediate and dramatic creating a seismic shift in the current therapeutic paradigm of periprosthetic joint infection. OBJECTIVES/GOALS: Periprosthetic joint infection (PJI) is a major cause of failure after joint replacement. Currently, the treatment of PJI relies on removing biofilm contaminated implants. Some of the bacteria within biofilm undergo a phenotypic shift becoming small colony variants (SCVs). SCVs induce local immunosuppression through PD-1/L1 signaling. METHODS/ STUDY POPULATION: We will infect cultured human macrophages and bone marrow aspirate with stable Staphylococcus aureus SVCs and treat with anti-PD-1 or anti-PD-L1 monoclonal antibodies with and without antibiotics (e.g., gentamycin, cefazolin, vancomycin, rifampicin) and assess the residual bacterial viability. We will utilize multiplexed ion beam imaging to quantify PD-1/L1 expression in human tissue from patients with a chronic PJI and compare those to patients undergoing an aseptic revision. Patients with a chronic PJI are likely to have increased expression of PD-1/L1 as their tissue samples are prospectively screened. RESULTS/ANTICIPATED RESULTS: SCVs reduce the phagocytic activity of macrophages and can survive intracellularly. SCVs also induce anti-inflammatory M2-macrophage polarization and recruit a heterogeneous group of immature monocytes and granulocytes called myeloid-derived suppressor cells (MDSC) to the periprosthetic microenvironment. M2macrophages and MDSCs then produce an immunosuppressive cytokine milieu characterized by increased IL-10 and decreased TNF- $\alpha$. Clinically isolated SCVs up-regulate the expression of PDL1 and PD-L2 on the surface of macrophages, representing a mechanism by which SCVs induce host immunosuppression and survive immune clearance. Our preliminary data show PD-L1 expression during septic PJI, but not in aseptic revisions. DISCUSSION/ SIGNIFICANCE OF FINDINGS: If immune checkpoint blockade is shown to increase bacterial clearance with or without antibiotics, host immunomodulation would represent a novel class of therapeutic adjuvants to assist surgical debridement and antibiotic administration that could be superimposed on existing treatment algorithms to improve PJI related outcomes.
REDUCED FRONTOSTRIATAL FUNCTIONAL CONNECTIVITY IN 41- TO 70-YEAR-OLD ADULTS WITH HIV*

Shiva Hassanzadeh-Behbahani ${ }^{1}$, Fan Nils Yang ${ }^{1}$, Margarita

Bronshteyn ${ }^{1}$, Matthew Dawson ${ }^{2}$, Princy Kumar ${ }^{1}$, John VanMeter ${ }^{1}$, David J. Moore ${ }^{2}$, Ronald J. Ellis ${ }^{2}$ and Xiong Jiang ${ }^{1}$

${ }^{1}$ Georgetown University Medical Center and ${ }^{2}$ University of California San Diego

ABSTRACT IMPACT: The knowledge acquired from my research can inform the development of early diagnostic methods for HIV-associated neurocognitive disorders. OBJECTIVES/GOALS: In the era of combination antiretroviral therapy (cART), the prevalence of HIV-associated neurocognitive disorders (HAND) remains high but the neural mechanisms are unclear. We examined whether older people with HIV (PWH) with minimal cognitive impairment have reduced functional connectivity in frontostriatal circuits compared to controls. METHODS/STUDY POPULATION: 99 PWH (mean age 56.6 years, 75\% male, 62\% Black, mean duration of HIV-infection 26.2 years $\pm 9.3,90 \%$ viral load $<50$ copies, $98 \%$ on stable cART) and 38 demographically-comparable controls (mean age 54.5 years, $71 \%$ male, $58 \%$ Black) participated in a cross-sectional study. A 7-domain neuropsychological battery and an Activities of Daily Living index were used to determine HAND diagnoses: 32 PWH met criteria for asymptomatic to mild HAND. Motor skill was assessed using the Grooved Pegboard Test by measuring performance speed. Structural MRI and resting-state functional MRI were collected. Seed-to-voxel analyses were conducted using 4 distinct regions in the striatum as seed regions. We used a voxel threshold of $\mathrm{p}<0.001$ and cluster threshold of $\mathrm{p}<0.05$ (FDR-corrected) after controlling for demographic variables. RESULTS/ANTICIPATED RESULTS: Compared to controls, PWH had lower resting state functional connectivity between the default mode region of the striatum (i.e., medial caudate) and bilateral superior frontal gyrus, supplementary motor cortex and paracingulate gyrus ( $\mathrm{p}<0.05$; cluster size: 567 voxels). Also, compared to controls, $\mathrm{PWH}$ had reduced resting state functional connectivity between the motor division of the striatum (i.e., posterior putamen) and anterior cingulate cortex and left supplementary motor cortex ( $\mathrm{p}<0.05$, cluster size: 405 voxels). Performance speed on the Grooved Pegboard motor test negatively correlated with functional connectivity between the motor region of the striatum and supplementary motor frontal regions in all participants (Spearman's rho=-0.18, $\mathrm{p}=0.04$ ). DISCUSSION/SIGNIFICANCE OF FINDINGS: Our results support the hypothesis that frontostriatal abnormalities are widely present in PWH and might play a key role in HAND development. Our data suggest that dysfunction within the frontostriatal circuits may be involved in motor impairment in $\mathrm{PWH}$, and ongoing inflammation may contribute to motor impairment and frontostriatal injury.

45724

vC2 Oncolytic Virotherapy Induces Robust Systemic Anti-Tumor Immunity and Increases Survival in an Immunocompetent B16F10-derived Mouse Melanoma Model Ifeanyi Kingsley Uche ${ }^{1}$, Natalie Fowlkes ${ }^{2}$, Luan $\mathrm{Vu}^{1}$, Tatiane Watanabe ${ }^{3}$, Mariano Carossino ${ }^{1}$, Rafiq $\mathrm{Nabi}^{1}$, Fabio Del Piero ${ }^{1}$, Jared S. Rudd ${ }^{1}$, Konstantin G. Kousoulas ${ }^{1}$ and Paul J.F. Rider ${ }^{1}$ ${ }^{1}$ Louisiana State University School of Veterinary Medicine, ${ }^{2}$ The University of Texas MD Anderson Cancer Center and ${ }^{3}$ North Carolina State University

ABSTRACT IMPACT: Our data demonstrate that VC2 oncolytic virotherapy has significant clinical potential. OBJECTIVES/ 